\title{
Comment on "Characterization of Interfacial Properties in Fiber-Reinforced Cementitious Composites"
}

\author{
Antoine E. Naaman and Jamil M. Alwan \\ Structural Division, Department of Civil and Environmental Engineering, University of Michigan, \\ Ann Arbor, Michigan $\mathbf{4 8 1 0 9}$
}

Modeling the process of pullout of a fiber from a matrix is a fundamental problem that has received great attention among researchers, as indeed shown by the review presented in the paper by Z. Li, B. Mobasher, and S. P. Shah. ${ }^{1}$ However, a number of recent studies have also addressed the specific problem of fiber pullout from a cementitious matrix and, as discussed below, contribute significantly to extending the current state of knowledge. ${ }^{2-6}$ In these references a fundamental study of the bond stress-slip relationship between steel fibers and cement composites is presented and correlated with the pullout problem. The analysis consists of a primal problem, whereby a complete pullout load versus slip curve can be predicted from an assumed (or experimental) bond stress versus slip relationship, and, a dual problem, in which the bond stress versus slip relationship is obtained from an experimental pullout curve. The solution presented is most general.

The main purpose of this comment is to show first that the model developed by Li et al. ${ }^{1}$ is a subset of the general model developed by Naaman et al. ${ }^{2-4}$ and second that, because of simplifying assumptions, it may be quite limited in scope.

\section{Particular Solution}

The approach used by Li et al. ${ }^{1}$ to model the fiber pullout process is basically a shear-lag approach, in which the matrix contribution to the axial deformation of the system is neglected. This is a subset of the general solution presented by Naaman et al., ${ }^{2.4}$ where the matrix deformation is accounted for. A proof is given next.

The shear-lag equilibrium equation is given by

$$
\frac{\partial \sigma_{t}(x)}{\partial x}=K(u-v)
$$

where $\sigma_{f}(x)$ is the axial stress in the fiber, $x$ the axial distance along the fiber axis, $u$ the local displacement of the fiber, $v$ the local displacement of the matrix, and $K$ the stiffness of the boundary shear-lag layer. Li $e t a l .{ }^{1}$ have eliminated the $v$ term from Eq. (1), leading to the solution given by Eq. (9) of their paper. It can be shown that Eq. (9) can be deduced from the general solution presented in Eq. (33) given by Naaman et al. ${ }^{2}$ (or Eq. (5.50) in Ref. 4), which is reproduced below:

$$
\begin{aligned}
Q=1 & +\frac{A_{\mathrm{m}} E_{\mathrm{m}}}{A_{\mathrm{f}} E_{\mathrm{f}}} \\
\Delta= & \left\{P(Q-1) u-\frac{t_{\mathrm{f}} u^{2}}{2}(Q-2)\right. \\
& \left.+\left(P-t_{\mathrm{f}} u\right) \operatorname{coth}[\lambda(l-u)] \frac{Q-2}{\lambda}-t_{\mathrm{f}} u l\right\} \mid A_{\mathrm{m}} E_{\mathrm{m}}
\end{aligned}
$$

\footnotetext{
S. M. Wiederhorn-contributing editor
}

Manuscript No. 195938C. Received March 2, 1992; approved December 4, 1992.
By simply assuming that $E_{\mathrm{m}}$ tends to infinity (i.e., an infinitely rigid matrix, or zero matrix deformation, as assumed by $\mathrm{Li}$ et al. $\left.{ }^{\prime}\right), \mathrm{Eq}$. (33) leads to

$$
\frac{Q-1}{A_{\mathrm{m}} E_{\mathrm{m}}}=\frac{Q-2}{A_{\mathrm{m}} E_{\mathrm{m}}}=\frac{1}{A_{\mathrm{f}} E_{\mathrm{f}}}
$$

Thus,

$$
\Delta=\frac{P-t_{\mathrm{f}} u}{\lambda A_{\mathrm{f}} E_{\mathrm{f}}} \operatorname{coth}[\lambda(l-u)]+\frac{\left(P-0.5 t_{\mathrm{f}} u\right) u}{A_{\mathrm{f}} E_{\mathrm{f}}}
$$

which matches Eq. (9) given in their paper, with the following corresponding notation: $u=a$ (debonded length), $l=L$ (embedded length), $t_{\mathrm{f}}=q_{\mathrm{f}}$ (frictional shear flow), $\Delta=U^{*}$ (fiber end displacement), and $\lambda=\omega=\left(K / A_{\mathrm{f}} E_{\mathrm{i}}\right)^{1 / 2}$.

Another drawback of the rigid matrix assumption is that it may lead to unrealistic simulation of the debonding mechanism along the fiber. Indeed a manipulation of $\mathrm{Eq}$. (1) of their paper and the shear-lag equation (Eq. (1) above) leads to the following results:

$$
\sigma_{i}(x)=\frac{P^{*}}{A_{\mathrm{f}}} \frac{\sinh (\omega x)}{\sinh (\omega L)}
$$

from which one can derive the interfacial shear stress as

$$
\begin{aligned}
\tau(x) & =\frac{r_{\mathrm{f}}}{2} \frac{\partial \sigma_{\mathrm{f}}(x)}{\partial x} \\
& =\frac{P^{*}}{2 \pi r_{\mathrm{f}}} \omega \frac{\cosh (\omega x)}{\sinh (\omega L)}
\end{aligned}
$$

Substituting $x=(L, 0)$ in Eq. (7) to compute the shear stress at both ends of the fiber, we get

$$
\begin{aligned}
\tau_{\text {face }} & =\frac{P^{*}}{2 \pi r_{\mathrm{f}}} \omega \operatorname{coth}(\omega L) \\
\tau_{\mathrm{cmb}} & =\frac{P^{*}}{2 \pi r_{\mathrm{r}}} \omega \operatorname{coth}(\omega L) \frac{1}{\cosh (\omega L)} \\
& =\tau_{\text {firce }} \frac{1}{\cosh (\omega L)}
\end{aligned}
$$

where $\cosh (\omega L) \geq 1$ for all positive values of $\omega L$. Therefore, $\tau_{\text {emb }}<\tau_{\text {face }}$, implying that debonding will always initiate at the point where the fiber penetrates the matrix. This result is not always correct. Indeed, an analytical study by Leong and $\mathrm{Li}^{7}$ has reported that, for the ratio $\alpha=V_{\mathrm{f}} E_{\mathrm{f}} / E_{\mathrm{c}}>0.5$, debonding will start at the embedded end of the fiber. This also agrees well with the analytical solution presented by Naaman et al. ${ }^{2,4}$ where $\tau_{\text {enb }}$ can exceed $\tau_{\text {face }}$ if

$$
P \lambda\left(A e^{\lambda \prime}-B e^{-\lambda \prime}\right)>P \lambda(A-B)
$$

or

$$
\frac{1}{Q}>0.5
$$


Table I. Predictive Results Computed Using Both Models

\begin{tabular}{|c|c|c|c|c|c|c|c|c|}
\hline \multirow[b]{2}{*}{ Sample $^{+}$} & \multirow[b]{2}{*}{$P / \Delta(\mathrm{N} / \mathrm{mm})$} & \multirow[b]{2}{*}{$P_{\text {peaki }}^{*}(\mathrm{~N})$} & \multirow[b]{2}{*}{$U_{\text {pil: }}^{*}(\mathrm{~mm})$} & \multirow[b]{2}{*}{$\begin{array}{c}a \\
(\mathrm{~mm})\end{array}$} & \multicolumn{2}{|c|}{$\tau_{y}(\mathrm{MPa})$} & \multicolumn{2}{|c|}{$\tau_{i}(\mathrm{MPa})$} \\
\hline & & & & & Liel al.' & $\begin{array}{l}\text { Naaman } \\
\text { et al } \stackrel{P .}{ }\end{array}$ & Li et $a .^{\prime}$ & $\begin{array}{l}\text { Naaman } \\
\text { el al. }\end{array}$ \\
\hline $\mathrm{H} 2 \mathrm{SF}$ & 7875 & 59.6 & 0.02 & 22.3 & 1.72 & 1.75 & 1.70 & 1.53 \\
\hline $\mathrm{H} 2 \mathrm{SL}$ & 12075 & 98.3 & 0.04 & 19.5 & 3.36 & 9.58 & 3.20 & 1.78 \\
\hline
\end{tabular}

${ }^{t}$ Fiber length $=25 \mathrm{~mm}(1 \mathrm{in}$.) and fiber diameter $=0.5 \mathrm{~mm}(0.019 \mathrm{in}$.).

where

$$
\frac{1}{Q}=\frac{A_{\mathrm{l}} E_{\mathrm{f}}}{A_{\mathrm{f}} E_{\mathrm{r}}+A_{\mathrm{m}} E_{\mathrm{m}}}=\alpha
$$

\section{Other Limitations}

Similar to the assumptions made by Naaman et al.,,$^{2.4}$ the model presented by Li et al.' characterizes the bond stress-slip relationship by three basic parameters: $\tau_{y}, \tau_{f}$, and $K$. However, it has been observed from an extensive investigation of pullout tests ${ }^{4}$ that the frictional shear stress at the fiber-matrix interface is a function of the local slip. To model such an observation, Naaman et al..$^{2.4}$ introduced a decay factor in their model, thus providing a solution more general than that presented in the paper by Li et al.

Furthermore, Li et al.' state that $\omega$ has to be computed first from their Eq. (9) for $a=0$, then substituted into their Eq. (14) along with $P_{\text {peak }}^{*}$ and $U_{\text {peak }}^{*}$ to solve for the debonded length, $a$. However, a restriction is made on $U_{\text {peak }}^{*}$, leaving a gap in the procedure, namely, should $U_{\text {peak }}^{*}$ exceed $U_{\text {crit }}^{*}, U_{\text {peak }}^{*}$ is to be limited to $U_{\text {crit }}^{*}$. Should a different fiber be investigated, a different numerical procedure is needed to solve directly for the three variables; for a similar case, Newton's algorithm for nonlinear systems has been followed by Naaman et al. ${ }^{3.4}$ and has led to good results.

The usefulness of a model is measured by how well it can simulate and predict experimentally observed results. Predictive results using both the model presented by Li et al.' and by Naaman et al.,2.4 are compared in Table I, using experimental data taken from Ref. 3. Only two specimens are used for illustration, a specimen $(\mathrm{H} 2 \mathrm{SF})$ where a steel fiber is pulled out from a plain cementitious matrix, and another, similar, specimen (H2SL) for which a latex emulsion was added to the matrix to improve the bond. Two observations can be made from Table I: (1) the rigid-matrix model presented by Li et al.' leads to slightly larger values of $\tau_{\mathrm{y}}$ because matrix deformations are neglected, and (2) in the case of specimen H2SL, where $U_{\text {peik }}^{*}>U_{\text {crit }}^{*}$, the model is insensitive to increased values of $\tau_{y^{*}}$ Indeed, the presence of latex leads to a maximum shear of about $9.58 \mathrm{MPa}(1390 \mathrm{psi})$, as supported by experiments, whereas the model of $\mathrm{Li}$ et al.' predicts only $3.36 \mathrm{MPa}(488$ psi).

\section{References}

Z. Li, B. Mobasher, and S. P. Shah, "Characterization of Interfacial Properties in Fiber-Reinforced Cementitious Composites,"J. Am. Ceram. Soc ., 74 [9] 2156-64 (1991).

A. E. Naaman, G. Namur, J. M. Alwan, and H. Najm, "Fiber Pullout and Bond Slip. I: Analytical Study," ASCE J. Struct. Eng., 117 [9]2769-90 (1991).

"A. E. Naaman, G. Namur, J. M. Alwan, and H. Najm, "Fiber Pullout and Bond Slip. Il: Experimental Validation." ASCE J. Struct. Eng., 117 [9] 2791$800(1991)$.

"A. E. Naaman, G. Namur, H. Najm, and J. M. Alwan, "Bond Mechanisms in Fiber-Reinforced Cement-Based Composites," Rept. No. UMCE 89-9, Department of Civil Engineering, University of Michigan, Ann Arbor, MI, Aug. 1989, AFOSR-TR-89-1276

'G. Namur and A. E. Naaman, "A Bond Stress Model for Fiber-Reinforced Concrete Based on Bond Stress Slip Relationship," ACI Mater. J., 86 [1] 45-57 (1989).

"G. Namur, A. E. Nalaman, and S. K. Clark, "Analytical Predication of PullOut Behavior of Steel Fibers in Cementitious Matrices." Mater. Res. Soc. Symp. Proc., 114, 217-24 (1988).

${ }^{7}$ C. K. Y. Leung and V. C. Li, "Applications of a Two-Way Debonding Theory to Short Fiber Composites," J. Cem. Compos., 21 [4] 305-17 (1990). 